\title{
CRITICAL CURRENT LIMITATIONS AND IRRADIATION EFFECTS IN HIGH-TEMPERATURE SUPERCONDUCTORS
}

\author{
A. Wiśniewski, R. Puźniak, M. Baran and H. Szymczak \\ Institute of Plysics, Polish Academy of Sciences \\ Al. Lotników 32/46, 02-668 Warszawa, Poland
}

\begin{abstract}
The discovery of high-temperature superconductors stimulated many groups of researchers to study properties of these materials. From the basic point of view the most important problem is to clarify the mechanism of high-temperature superconductivity. From the practical point of view the most challenging task is to find mechanisms of strong pinning of the vortices, and thereby to obtain materials with high critical current density. The aim of this paper is to discuss problems connected with critical current limitations and to present possibilities which offer irradiation effects with respect to critical current enhancement.
\end{abstract}

PACS numbers: 74.60.Ge, 74.60.Jg, 61.80.Hg

\section{Mechanisms of critical current limitations}

In principle, there are three mechanisms which limit the critical current density, $j_{\mathrm{c}}$, in type II superconductors: depairing, decoupling, and depinning.

\subsection{Depairing}

Depairing critical current means a value of current for which the kinetic energy of supercurrent carriers is equal to the condensation energy in the superconducting state. In other words, for this value of current the superelectrons are excited over the superconducting gap and the Cooper pairs break up. According to the BCS theory the depairing critical current is inversely proportional to the coherence length $\xi$. In high temperature superconductors (IITSC), due to a very small value of $\xi$, this current is quite large $\left(10^{9} \mathrm{~A} / \mathrm{cm}^{2}\right.$ at low temperatures), therefore from the practical point of view this process is not the real limitation. 


\subsection{Decoupling}

The critical current density, $j_{\mathrm{c}}$, is reduced drastically by layers of non-superconducting material. For layers consisting of insulators the supercurrent could be maintained by tunneling, for semiconductor or normal metal layer the proximity effect could enable one a flow of supercurrent. The critical current limited by decoupling is very sensitive to the external magnetic field. The magnetic field of the order of ten Gauss can lead to one order of magnitude reduction of $j_{\mathrm{c}}$. This mechanism is the most evident in IITSC ceramics. In ceramic samples, prepared in the conventional manner, the so-called intragrain critical current (the current that could flow through the grain) is 3 or 4 orders of magnitude higher than the intergrain current, i.e. the current which could flow through the whole sample. However, careful examination of $j_{\mathrm{c}}$ as a function of external magnetic field in HTSC single crystals shows that mechanism of decoupling can be responsible for $j_{\mathrm{c}}(H)$ behavior. This problem will be discussed further.

\subsection{Depinning}

Above the first critical field the magnetic flux penetrates a sample in a form of flux lines (vortices) and each vortex carries one flux quantum $\Phi_{0}$. The transport current flowing through the sample exerts the Lorentz force acting on each flux line

$$
\boldsymbol{F}_{\mathrm{L}}=\boldsymbol{J} \times \boldsymbol{B} .
$$

The Lorentz force can accelerate a flux line to a velocity $v$ and as a result an electric field $\boldsymbol{E}=\boldsymbol{B} \times \boldsymbol{v}$ occurs in the superconductor, which becomes resistive. Also in the case, when there is no external magnetic field, the field generated by the transport current enters the sample as flux lines. The only way to obtain reasonable critical currents is to make the movement of the flux lines impossible. This can be achieved by defects existing in the material. In the neighborhood of the defects, the critical temperature and the Ginzburg-Landau parameter $\kappa$ are different from those for the rest of the material. For this reason, the condensation energy of such regions is reduced. When the normal core of a flux line is located at a defect, the so-called pinning force $\boldsymbol{F}_{\mathrm{P}}$ makes impossible a movement of the flux line as long as it is bigger than $\boldsymbol{F}_{\mathrm{L}}$. This type of pinning is called the core pinning. Another possible mechanism is connected with magnetic interactions between flux lines and defects or sample surface. It is called the magnetic pinning. The characteristic length for magnetic pinning is the magnetic penetration depth $\lambda$. Since the vortex core radius is equal to the colherence length $\xi$, its value is the characteristic length for the core pinning.

Flux lines can be also hindered by mutual interactions. The critical current is proportional to the pinning force and is defined by the balance between the pinning and Lorentz force

$$
\boldsymbol{F}_{\mathrm{L}}=J_{\mathrm{c}} \times \boldsymbol{B}=\boldsymbol{F}_{\mathrm{P}} \text {. }
$$

Mechanisms of the above-mentioned critical current limitations concern all type II superconductors [1]. It seems wortliwhile to point out some problems that 
are specific for HTSC materials, especially when they are expected to work at higher temperature (e.g. $77 \mathrm{~K}$ ).

\section{The main peculiarities of HTSC}

From the point of view of critical currents limitations the following characteristic features of IITSC compounds seem to be relevant: a small value of the coherence length $\xi$, a large value of the penetration depth $\lambda$, high anisotropy of the crystal structure and physical properties, relatively high critical temperature, an irreversibility line (or melting line).

\subsection{A small value of the coherence length $\xi$}

For $\mathrm{YBa}_{2} \mathrm{Cu}_{3} \mathrm{O}_{7}, \xi_{a b} \approx 12 \AA, \xi_{\mathrm{c}} \approx 3-4 \AA$, for $\mathrm{Bi}_{2} \mathrm{Sr}_{2} \mathrm{CaCu}_{2} \mathrm{O}_{8}, \xi_{a b} \approx 30 \AA$ and $\xi_{c} \approx 1 \AA$. The symbols $\xi_{a b}$ and $\xi_{\mathrm{c}}$ denote the coherence length in the $a-b$ plane and in the $c$-direction, respectively. According to the existing experimental data, anisotropy in the $a-b$ plane is negligible, therefore $\xi_{a b}$ corresponds to $\xi_{a} \approx \xi_{b}$. In contrast, the coherence length of conventional metallic superconductors ranges from 100 to $600 \AA$. The coherence length $\xi$ is the fundamental parameter, and its value strongly influences many quantities describing processes involved in critical current limitations. In more detail it will be discussed below. It should be emphasized that the tendency to form the Josephson weak links (particularly at the grain boundaries) is just a consequence of small value of $\xi$. The fact that $\xi$ along the $c$-axis is much shorter than the length of the crystallographic axis $c(c \approx 11.7 \AA$ in $\mathrm{YBa}_{2} \mathrm{Cu}_{3} \mathrm{O}_{7}$ ) yields a possibility of a specific mechanism of pinning, the so-called intrinsic pinning [2]. According to Tachiki and Takahashi [2] the layered structure of HTSC itself works as a strong pinning center of the vortices. This mechanism for $\mathrm{YBaCuO}$ could be explained as follows. The $\mathrm{CuO}_{2}$ layers and their surrounding are strongly superconducting. The layers containing $\mathrm{CuO}$ chains and their surrounding are weakly superconducting and characterized by a smaller superconducting order parameter. Let us consider a situation when the field (vortex) direction is parallel to the $a-b$ plane. The superconducting order parameter inside the core of vortex almost vanishes. Therefore, the vortex placed around the layers with $\mathrm{CuO}$ chains realizes the minimum of energy. Consequently, the vortices are stabilized in these planes. For the field at a small angle with respect to the $a-b$ plane, the vortex cores keep the position as above at the greater part of their length, turning and crossing the planes at some points as required by the macroscopic flux direction. When the flux line cross the $a-b$ planes, it may be viewed as being composed of $2 \mathrm{D}$ vortices (called by Clem the "pancake vortices" [3]) localized in individual layers and lateral segments in the interlayer spacings. The magnetic field perpendicular to the $a-b$ planes produces also the "pancake" vortices that form stacks along the field direction, interacting by the Josephison coupling. The thermal excitations can easily decouple vortices from neighboring planes. The mechanism of the intrinsic pinning well explains experimental results obtained for $\mathrm{YBaCuO}$ epitaxial thin films. For the current flowing in the $a-b$ plane the highest $j_{\mathrm{c}}$ value is realized with the external magnetic field parallel to the $a-b$ plane. For the magnetic field parallel 
to the $c$-axis $j_{\mathrm{c}}$ becomes lower [4]. Another consequence of the small value of $\boldsymbol{\xi}$ is a possibility of a contribution of the point defects (e.g. missing atoms) to the pinning.

\subsection{A large value of the penetration depth $\lambda$}

For $\mathrm{YBa}_{2} \mathrm{Cu}_{3} \mathrm{O}_{7}, \lambda_{a b} \approx 1400 \AA, \lambda_{c} \approx 7000 \AA$ and for $\mathrm{Bi}_{2} \mathrm{Sr}_{2} \mathrm{CaCu}_{2} \mathrm{O}_{8}$ respective values are equal to about $3400 \AA$ and $7500 \AA$. Therefore, the internal magnetic field in the vortex lattice is almost uniform, so the magnetic pinning is very weak. The intervortex forces caused by the current-current interactions of the vortices are also relatively weak (e.g. in comparison with the intrinsic pinning) for the same reason.

\subsection{High anisotropy of the cryslal structure and physical properties}

The structure of IITSC compounds can be regarded as a stack of $\mathrm{CuO}_{2}$ planes (parallel to the basal $a-b$ plane) being quasi two-dimensional metal sheets weakly coupled with adjacent planes. The character of electric conductivity, in the normal state, along the $a-b$ plane is of metal type, and in the $c$-direction is of semiconducting type (single particle tunneling). Strong anisotropy is exhibited by basic parameters such as the coherence length, the penetration depth, the critical current as well as the first and second critical fields. The strong anisotropy has been found in the best known IITSC compound, $\mathrm{YBa}_{2} \mathrm{Cu}_{3} \mathrm{O}_{7-x}$. Because of the increase in the distance between the $\mathrm{CuO}_{2}$ planes and the decrease in the coherence length $\xi$ in the $c$-direction, in $\mathrm{BiSrCaCuO}$ and $\mathrm{TlBaCaCuO}$ compounds in comparison with $\mathrm{YBaCuO}$, the anisotropy is stronger by one order of magnitude. Because of the scope of this paper, the anisotropy of the critical current density are discussed below in more details.

Generally, $j_{\mathrm{c}}$ should depend not only on the anisotropy of material properties but also on the possible anisotropy of pinning forces. Therefore, two factors - the direction of external magnetic field and the direction of current - should be taken into account. In the case of external magnetic field along the $c$-axis an essentially isotropic critical current is induced in the $a-b$ plane. According to the notation proposed by Malozemof [5] it can be denoted by $j_{c}^{a b, c}$, where the first superscript $a b$ denotes the current orientation, and the second one $c$ denotes the field direction. When the field is parallel to the $a-b$ plane, two components should be distinguished: $j_{c}^{c, a b}$ component for the current in the $c$-direction, and $j_{c}^{a b, a b}$ component for the current along the $a-b$ plane. The corresponding Lorentz forces act along the $a-b$ plane and perpendicular to the $a-b$ plane, respectively. Because of the already mentioned intrinsic pinning, $j_{\mathfrak{c}}^{a b, a b}$ is at least one order of magnitude greater than $j_{c}^{c, a b}$. The careful magnetic measurements of all these three components [6] show that $j_{\mathrm{c}}^{a b, c}$ is close to $j_{\mathrm{c}}^{a b, a b}$.

\subsection{Relatively high critical temperature}

HTSC can be used at the temperatures at least one order of magnitude higher than conventional superconductors. Therefore, the influence of the thermal 
activation is much more pronounced in these materials [7, 8]. At sufficiently high temperatures the thermal energy, $k_{\mathrm{B}} T$, is comparable with the flux pinning energy. As a result, thermally assisted motion of flux lines occurs, leading to the energy dissipation. One can distinguish the flux creep [9] - hopping from one potential pinning well to another, and viscous thermally assisted flux flow (TAFF) [10]. The flux creep can be detected as a decay of magnetization (superconducting current) with time and the flux-flow as an appearance of resistivity when an electric current is passing through a superconductor in a magnetic field. In fact, large magnetic relaxation, approximately logarithmic in time, is one of the characteristic features of HTSC compounds. The logarithmic relaxation rate $\mathrm{d} M / \mathrm{d} \ln (t)$ shows a peak, both as a function of temperature and of magnetic field. The thermally assisted flux-flow is characterized by linear current-voltage dependence with the resistivity $\rho_{\text {TAFF }}$ strongly dependent on temperature. TAFF well explains large broadening of $\rho(T)$ in magnetic field, commonly observed in IITSC. This broadening is anisotropic, being greater for the field parallel to the $c$-axis [11]. The thermally assisted flux-flow occurs even for values of current much lower than $j_{\mathrm{c}}$. The flux creep occurs only for currents on the level of $j_{c}$, when the superconductor is in the critical state. In the conventional (type II) superconductors this effect is observed at temperatures very close to $T_{\mathrm{c}}$. In IITSC, the giant flux creep [12] occurs in large temperature range.

The thermal activation of a vortex situated at the pinning center gives a certain probability of the vortex transition to an excited state. Discussing this problem, one can describe a pinning center as a potential well of $U_{0}$ depth. The value of $U_{0}$ is proportional to $V H_{\mathrm{c}}^{2}$, where $H_{\mathrm{c}}$ means the thermodynamical critical field and $V$ - a characteristic volume of vortex interaction with the pinning center. This volume is determined by the value of $\xi$. Because the coherence length in ITSC is very small, in spite of relatively high value of $H_{c}$, the value of $U_{0}$ is rather small. The values of $U_{0}$ in IITSC are of one or two orders of magnitude smaller than those in typical conventional superconductors, where they attain several electronovolts. In $\mathrm{YBaCuO}$ single crystals, according to the data presented by Yeshurun et al. [13], $U_{0} \approx 0.02 \mathrm{eV}$ for the magnetic field parallel to the $c$-axis and $U_{0} \approx 0.2 \mathrm{eV}$ for the field parallel to the $a-b$ plane. This is the reason why the thermal activation at high temperatures strongly limits possibilities of obtaining high values of critical current. The interesting possibility was suggested by Blatter et al. [14] and by Motta and at al. [15]. Flux lines may still move even at very low temperatures, where a thermal activation rate becomes negligibly small, due to the quantum mechanical tunneling.

\subsection{An irreversibility line (or melling line)}

In HTSC materials the onset points of the diamagnetism and of the superconducting critical current form apparently different lines in the $H-T$ plane, i.e. the phase transition line and the irreversibility line, respectively. Above the irreversibility line the reversible magnetization is observed only, whereas below it, the magnetization becomes increasingly irreversible. This line is sometimes called the "melting line", because it is assumed that above this line the long range vortex order is lost, and the regular Abrikosov array of vortices is "melted". The 
irreversibility line (onset of $j_{\mathrm{c}}$ ) shifts a region of potential applications to lower fields and temperatures than that resulting from the phase transition line $H_{\mathrm{c} 2}(T)$. For $\mathrm{YBa}_{2} \mathrm{Cu}_{3} \mathrm{O}_{7}$, onset of $j_{\mathrm{c}}$ is about $5 \mathrm{~K}$ below $T_{\mathrm{c}}$ in the field of few Tesla. In the case of bismuth based compounds this effect is much more pronounced. The respective shift of about $50 \mathrm{~K}$, even in the field of few $\mathrm{kOe}$, was observed in $\mathrm{Bi}_{2} \mathrm{Sr}_{2} \mathrm{CaCu}_{2} \mathrm{O}_{8+x}\left(T_{\mathrm{c}}=85 \mathrm{~K}\right)$. It completely rules out this compound from the applications at $77 \mathrm{~K}$. The irreversibility lines in IITSC are anisotropic and always occur at higher fields and temperatures for external magnetic field parallel to the $a-b$ plane. This fact can be easily explained within the framework of intrinsic pinning mechanism anisotropy. It was believed that the temperature region of reversible flux motion in conventional metallic superconductors would be too small to be easily observed and of any significance. Ilowever, the existence of surprisingly large region of reversible magnetization was found recently for multifilamentary $\mathrm{Nb}_{3} \mathrm{Sn}$ and $\mathrm{Nb}-\mathrm{Ti}$ wires [16].

The mechanisms of the critical current limitations, discussed above, can be summarized as follows. The dissipation of energy in the mixed state, arising from the motion of flux lines, is described by a competition among the driving force, pinning force and a force exerted by other flux lines. The driving force acting on every flux line - it is the Lorentz force $J \times B$. The pinning force acting on every flux line has its source in defects originally existing in the material or artificially introduced. The competition among these forces depends on temperature as well as on strength and orientation of the magnetic field. When flux lines are parallel to the $a-b$ plane the intrinsic pinning plays an important role.

\section{Characteristic features of various types of HTSC materials}

Values of critical current in IITSC compounds are strongly dependent on the type of the material. Different values of $j_{c}$ are obtained for a single crystal, epitaxial film or polycrystalline samples. For this reason, it seems worthwhile to summarize shortly typical properties of each type. This will be done below, mainly for $\mathrm{YBa}_{2} \mathrm{Cu}_{3} \mathrm{O}_{7-x}$. However, many of these features are characteristic for other compounds as well.

\subsection{Single cryslals}

Typical values of $j_{c}$ in the $a-b$ plane for $\mathrm{YBa}_{2} \mathrm{Cu}_{3} \mathrm{O}_{7-x}$ at $77 \mathrm{~K}$ and in a field of about $10 \mathrm{kOe}$ are of the order of $10^{4} \mathrm{~A} / \mathrm{cm}^{2}$. This value is limited by depinning and it can be significantly enhanced (even more than one order of magnitude), e.g. by neutron irradiation [17]. In majority of $\mathrm{YBa}_{2} \mathrm{Cu}_{3} \mathrm{O}_{7-x}$ single crystals the twin boundaries are present that can act as pinning centers. Ilowever, there is no consensus on the relative strength of twin pinning in comparison to other sources of pinning. The characteristic feature of single crystals of $\mathrm{YBa}_{2} \mathrm{Cu}_{3} \mathrm{O}_{7-x}$, also observed in some ceramic samples, is the so-called "fish tail" effect. As was shown by Daeumling et al. [18], in spite of the first maximum in a magnetization loop in almost zero field, an anomalous second large peak was observed, e.g. at $70 \mathrm{~K}$ in about $45 \mathrm{kOe}$. This effect was explained, assuming existence of oxygen-deficient 
regions with significantly lower $H_{\mathrm{c} 2}(T)$, where superconductivity is suppressed at higher fields. These regions can play a role of very efficient pinning centers. An appearance of a steep fall beyond the second peak indicates that presumably these regions form internal barriers leading to some kind of crystal granularity [19]. In this case, it is not an easy task to calculate properly $j_{\mathrm{c}}$ from the magnetization measurements because of uncertainty concerning the value that should be used as a radius of a supercurrent loop. Single crystals play an important role in studies of basic properties of IITSC materials but their applications seem to be strongly limited because of small sizes (less than $0.1 \mathrm{~cm}^{3}$ ).

\subsection{Epilaxial thin films}

The highest $j_{\mathrm{c}}$ values were obtained in these materials. Many groups of researchers achieved $j_{\mathrm{c}}$ of the order of $10^{6} \mathrm{~A} / \mathrm{cm}^{2}$ at $77 \mathrm{~K}$ in $\mathrm{YBa}_{2} \mathrm{Cu}_{3} \mathrm{O}_{7-x}$ films. The critical current density in thin films, limited by depinning as in single crystals, gives a possibility of $j_{\mathrm{c}}$ enhancement by irradiation. Measurements of angular dependence of $j_{\mathrm{c}}(B)$ for the magnetic field parallel to the $a-b$ plane give evidence of intrinsic pinning in thin films. Iowever, this mechanism cannot explain the high values of $j_{c}$ for the field parallel to the $c$-axis. Investigations of $\mathrm{YBaCuO}$ thin films, by means of scanning tunneling microscopy [20], have shown a large density of screw dislocations, which presumably play a role of very effective pinning centers, and are responsible for high values of $j_{c}$.

\subsection{Polycryslalline samples}

The values of $j_{\mathrm{c}}$ in ceramic samples, produced by conventional methods, are two to four orders of magnitude lower than those of single crystals or epitaxial thin films. The basic reasons for that fact are following: atomic disorder at grain boundaries, random orientation of grains, stoichiometry imperfections, secondary phase at grain boundaries, and poor connections between grains. All the above reasons, because of short coherence length and large anisotropy of IITSC, lead to a weak coupling at the grain boundaries between strongly superconducting grains. This weak-link behavior results in a low transport current density in zero magnetic field, with typical values of order of $100 \mathrm{~A} / \mathrm{cm}^{2}$ at $77 \mathrm{~K}$, and with a strong decrease in $j_{\mathrm{c}}$ in magnetic field of tens of Gauss. As a natural solution of the problems discussed above, several methods were proposed to achieve higher $j_{c}$ value: grain alignment, improvement by additives, melt-texturing.

\subsubsection{Grain alignment}

Using mechanical texturing, magnetic orientation and directional solidification one aligns grains within a few degrees, thereby the current can flow along strongly superconducting $a-b$ planes. In magnetic ordering method, for example, a powdered sample is mixed with a solvent and oriented in a magnetic field of several Tesla. Then the solvent is evaporated, the field is removed and the sample is sintered. This method enables one to obtain samples with $j_{\mathrm{c}} \approx 2000 \mathrm{~A} / \mathrm{cm}^{2}$ at 77 in $[21]$. 


\subsubsection{Improvement by addilives}

Many groups of scientists have studied the influence of different additives (silver, gold, ferromagnetic elements) on inter- and intragrain critical current density. It seems that the most promising results were obtained with silver. This element, depending on the processing technology, either forms precipitates at the grain boundaries or substitutes copper in superconducting compound. An overwiev of effects of silver addition is given by Schinde et al. [22]. Silver placed within grain boundaries leads to better connections between grains and to closer packing. As a result $j_{c}$ of order of $10^{4} \mathrm{~A} / \mathrm{cm}^{2}$ can be achieved at $77 \mathrm{~K}$ and in zero magnetic field. Silver introduced to the compound enhanced the intragrain critical current 5 times. Another important possibility was reported by Schilling et al. [23]. An addition of $\mathrm{BaTiO}_{3}(5 \mathrm{w} \%)$ to $\mathrm{YBa}_{2} \mathrm{Cu}_{3} \mathrm{O}_{7-x}$ completely changed the field dependence of $j_{\mathrm{c}}$. As a result, an improvement by one order of magnitude was achieved at $77 \mathrm{~K}$ in $40 \mathrm{kOe}$, in comparison with a pure sample.

\subsubsection{Mell-texturing}

In the authors' opinion it is the most promising method for obtaining samples with high $j_{\mathrm{c}}$ suitable for practical applications. This method, originally introduced by Jin et al. [24], was called melt-textured growth (MTG). Some other approaches were proposed by Salama et al. [25] - called liquid-phase processing (LPP), Murakami et al. [26] - melt powder melt growth (MPMG) and by Zhou Lian et al. [27] - powder melting process (PMP). In all these processes, very dense and well-aligned samples with large grain boundary areas and grains up to a centimeter in length are obtained. It can be observed from the phase diagram of $\mathrm{YBa}_{2} \mathrm{Cu}_{3} \mathrm{O}_{7-x}$ (123) [28] that the sample heated above the peritectic decomposition temperature $\left(1015^{\circ} \mathrm{C}\right)$ melts incongruently into a liquid, the solid $\mathrm{Y}_{2} \mathrm{BaCuO}_{5}$ (211) and the solid $\mathrm{Y}_{2} \mathrm{O}_{3}$ phases according to the reactions:

$$
\begin{array}{ll}
123 \rightarrow 211+\text { Liquid, } & 1015^{\circ} \mathrm{C}<T<1300^{\circ} \mathrm{C}, \\
211+\text { Liquid } \rightarrow \mathrm{Y}_{2} \mathrm{O}_{3}+\text { Liquid, } & 1300^{\circ} \mathrm{C}<T<1500^{\circ} \mathrm{C} .
\end{array}
$$

On subsequent slow cooling of the semi-solid melt below the peritectic temperature (in some approaches the temperature gradient is used to enhance grains alignment) oriented 123 samples are formed, with 211 inclusions randomly distributed throughout the superconducting matrix. Critical current densities of such samples approach to $10^{5} \mathrm{~A} / \mathrm{cm}^{2}$ in zero magnetic field and more than $10^{4} \mathrm{~A} / \mathrm{cm}^{2}$ in $10 \mathrm{kOe}$. Although the size of 211 inclusions is relatively large (about 1 micron), in comparison with the coherence length, they form very efficient pinning centers. The important advantage, as it was pointed out by Murakami et al. [29], is the fact that the flux creep rate is quite small in comparison with that of thin films. It means that the pinning potentials of large normal precipitations are much bigger than those of small pinning centers. Therefore, the flux motion is less affected by thermal activation. In fact, in the case of the point defects of the order of the coherence length, the pinning energy is described as follows:

$$
U_{\mathrm{p}}=\left(H_{\mathrm{c}}^{2} / 8 \pi\right)(4 / 3) \pi \xi^{3} .
$$

At the temperature of $77 \mathrm{~K}$, when $H_{\mathrm{c}} \approx 3000 \mathrm{Gs}$ and $\xi \approx 20 \AA$, the pinning energy is equal to about $0.01 \mathrm{eV}$, though it is of the order of thermal energy $k_{\mathrm{B}} T$. In the 
case of larger defects, the pinning energy is described by the following formula:

$$
U_{\mathrm{p}}=\left(H_{\mathrm{c}}^{2} / 8 \pi\right) \pi \xi^{2} d
$$

where $d$ is a length of defect. Taking typical size of 211 inclusions, $d \approx 1 \mu \mathrm{m}$, one can get $U_{\mathrm{p}} \approx 5 \mathrm{eV}$.

One should remember that from the point of view of practical applications the problem of availability of superconductors in the form of relatively long wire or tape is important. In such wires critical densities of order of $10^{5} \mathrm{~A} / \mathrm{cm}^{2}$ should be achieved. At present, the most encouraging results were obtained for silver coated $\mathrm{Bi}_{2} \mathrm{Sr}_{2} \mathrm{Ca}_{2} \mathrm{Cu}_{3} \mathrm{O}_{10}\left(T_{\mathrm{c}}=105 \mathrm{~K}\right)$ wires. They are characterized by extensive texturing and small-angle grain boundaries. In $50 \mathrm{~cm}$ long wires, $j_{\mathrm{c}} \approx 10^{4} \mathrm{~A} / \mathrm{cm}^{2}$ was achieved at $77 \mathrm{~K}$, in the field of $10 \mathrm{kOe}$ applied parallel to the wire [30]. In multifilamentary $\mathrm{YBa}_{2} \mathrm{Cu}_{3} \mathrm{O}_{x}$ wires, 20 meters long, $j_{\mathrm{c}} \approx 4000-6000 \mathrm{~A} / \mathrm{cm}^{2}$ was achieved at $50 \mathrm{~K}$ in zero magnetic field [31]. Technical applications of conventional superconductors have been realized with $\mathrm{Nb}-\mathrm{Ti}$ alloys $\left(T_{\mathrm{c}}=9 \mathrm{~K}\right)$ and $\mathrm{Nb}_{3} \mathrm{Sn}$ $\left(T_{\mathrm{c}}=18 \mathrm{~K}\right)$. In $\mathrm{Nb}-\mathrm{Ti}$ and $\mathrm{Nb}_{3} \mathrm{Sn}$, fabricated in the form of multifilamentary conductors, critical transport current densities exceeding $10^{5} \mathrm{~A} / \mathrm{cm}^{2}$ at $4.2 \mathrm{~K}$ in the fields of about $100 \mathrm{kOe}$ can be achieved.

\section{Measurement techniques}

In order to characterize IITSC materials in details, various measurement techniques should be used. Ilowever, basic parameters, such as critical temperatures, critical fields and critical currents, are determined by two main methods transport measurements and magnetic measurements (both ac susceptibility and dc magnetization).

The critical current, which is the subject of particular interest of the paper, can be determined from transport and magnetic measurements as well. However, one should be careful comparing $j_{\mathrm{c}}$ values obtained by different methods. Even for the same sample $j_{\mathrm{c}}$ values frequently differ by a factor of 3-4.

In the transport measurements the four-probe method is generally in use, and the critical current is defined as a value of current for which non-zero resistance of a sample appears. The precision of such measurements depends on the sensitivity and the resolution of measuring set being applied. In typical equipment the criterion of $1 \mu \mathrm{V} / \mathrm{cm}$ is fulfilled. The crucial problem for this method is also the quality of contacts. Especially current contacts are important, because of possible dissipation of energy in these contacts in the case of high current density. In the case of very high critical current density the pulse method is commonly in use. It lowers an average current flowing through the sample, however proper determination of $j_{\mathrm{c}}$ becomes difficult. Also the geometry of the investigated sample and the geometry of contacts, the latter especially important in the case of small samples, can be a source of some errors in evaluation of $j_{\mathrm{c}}$.

The magnetic method of $j_{\mathrm{c}}$ determination as a contactless method is very suitable from the experimental point of view. The value of $j_{\mathrm{c}}$ is determined from the magnetic hysteresis loop. In this method the most important problem is the question: to what extent the theoretical model used in the calculation of $j_{\mathrm{c}}$ describes the real physical situation. The most commonly in use is the Bean model 
of the critical state $[32,33]$ and also some more sophisticated models based on this first model (e.g. the critical current anisotropy model [6]). One should take into account that the sensitivity of magnetic measurements for samples of millimeter sizes is about three orders of magnitude better than that commonly obtained from the transport measurements. In the case of high values of the critical current, when one compares the values obtained from the magnetic method with transport pulse method, the following facts should be taken into account. The time scale for the transport method depends on the time of pulse. Typically it is $10^{-3} \mathrm{~s}$, which is the time scale of quite large magnetic moment relaxation. For the time scale of $M(H)$ loop measurement of $10^{2} \mathrm{~s}$ and logarithmic time dependence of relaxation the critical current delay is much smaller (about five times). In any case the value of magnetic $j_{\mathrm{c}}$ determined from $M(I I)$ loop should be smaller than $j_{\mathrm{c}}$ obtained from the transport pulse method and should correspond to the real critical current density.

For all these reasons the values of $j_{\mathrm{c}}$ should be treated as rather approximate numbers, especially when one compares results obtained by different techniques and on different samples (single crystals, thin films, ceramics).

\section{Irradiation effects in HTSC materials}

Understanding of the mechanisms that control the critical current density $j_{\mathrm{c}}$ is not satisfactory, even in the case of conventional type II superconductors. The situation seems to be more complicated for IITSC compounds, because each defect with the size comparable to the coherence length $\xi$ can play the role of a pinning center, and therefore change the value of $j_{\mathrm{c}}$. $A s \xi$ is of the order of nanometers or less, a large variety of structural defects could contribute to the pinning process. Moreover, $\xi$ is highly anisotropic and temperature dependent and it introduces additional complications.

It has been established that the defects introduced by neutron, proton or heavy ion irradiation are eflective pinning centers. The irradiation leads to significant enhancement of $j_{\mathrm{c}}$, modifying also the irreversibility line and the value of the activation energy $U_{0}$ [34]. Ilowever, it should be pointed out, especially with regard to the $j_{c}$ enhancement that the effect depends strongly on the type of material (single crystal, ceramic or thin film). In the case of typical ceramics, with $j_{c}$ limited by weak links, the irradiation enlances the flux pinning inside the grains, but simultaneously damages the intergrain junctions. In consequence, the transport critical current decreases after irradiation [35-37]. Ilence, the promising materials for application are epitaxial thin films, single crystals and melt-textured materials. In those materials weak links are absent or they do not limit $j_{\mathrm{c}}$. On the other hand, the application of various linds of irradiation helps to improve understanding the nature of the pinning centers and all related phenomena. The following differences should be taken into account, when one compares results of irradiation with various kinds of particles. Ncutrons can easily penetrate the whole volume of big samples because of their lack of an electric charge. The main result of irradiation with fast neutrons (energies of the order of $\mathrm{MeV}$ ) is a production of defect cascades with a mean diametcr of about $5 \mathrm{~nm}$. It is close to the flux line 
diameter of $2 \xi_{a b}=7.6 \mathrm{~nm}$ at $77 \mathrm{~K}$ for $\mathrm{YBaCuO}$. A certain disadvantage of this kind of irradiation is the activation of the material by thermal and epithermal neutrons. This fact excludes compounds with elements of long decay time. Generally, the samples can be measured safely after some time, depending on the compound and applied neutron dose. For $\mathrm{YBaCuO}$ or $\mathrm{BiSr} \mathrm{CaCuO}$ compounds this time is not too long for moderate doses, and usually it is not more than a few weeks.

In principle, the majority of neutron irradiation experiments have been made with fast neutrons. However other possibilities are not excluded. Fleischer et al. [38] studied the effect of fission fragments on critical intragrain currents in $\mathrm{YBaCuO}$ ceramic. The samples were sintered with natural uranium and fission was induced by exposure to thermal neutrons. An increase in intragrain current in the doped samples was observed, while in pure samples $j_{\mathrm{c}}$ was almost unchanged. It is also interesting to explore the possibilities of flux pinning enhancement in Li-doped materials following thermal neutron irradiation through the reaction products ${ }^{3} \mathrm{H}$ $(2.73 \mathrm{MeV})$ and ${ }^{4} \mathrm{IIe}(2.05 \mathrm{MeV})$ [39]. In the particular case of $\mathrm{GdBaCuO}$, because of large neutron capture cross section of ${ }^{155} \mathrm{Gd}$ and ${ }^{157} \mathrm{Gd}$, thermal neutron irradiation can lead to the creation of defects different from those due to fast neutron induced cascades [40].

Heavy ion irradiation leads to the formation of continuous latent tracks, i.e. tubes of amorphous material of about $7 \mathrm{~nm}$ in diameter. IIardy et al. [41] found that latent tracks produced by $5.3 \mathrm{GeV} \mathrm{Pb}$ ions induced a strong anisotropic flux pinning and critical current density. It results in twentyfold increase in $j_{\mathrm{c}}$ at $77 \mathrm{~K}$ in the magnetic field of $20 \mathrm{kOe}$ oriented parallel to the tracks, for the fluence of $3 \times 10^{11} \mathrm{ions} / \mathrm{cm}^{2}$. In this case the pinning energy of $0.5 \mathrm{eV}$ of irradiated material was inferred. This kind of irradiation does not activate the material. However, the application of the method is limited by the ions penetration depth of about $200 \mu \mathrm{m}$, depending on the energy.

Proton irradiation produces mainly point defects and does not activate materials. The penetration depth of protons is also limited to about $200 \mu \mathrm{m}$. Furthermore, proton irradiation facilities allow one to use collimated beams (diameter of about 0.3 microns). It provides a very convenient possibility of modulation of the superconducting properties of thin films, including a production of non-superconducting regions. This possibility seems to be very important with regard to a production of small scale devices based on IITSC compounds. Another advantage of ion and proton irradiation is the possibility of applying the particle beam in defined directions with respect to the crystallographic axes. This aspect is important because of the high anisotropy of IITSC compounds.

One can notice the difference between neutron, heavy ions and proton irradiations by the comparison of their influence on the position of the irreversibility line and magnetic relaxation. Measurements of proton irradiated single crystals of $\mathrm{YBaCuO}$ [42] show a large increase in $j_{c}$, but no change of the irreversibility line. On the contrary, 5.3 GeV Pb ions irradiation [43] leads to a dramatic change of the irreversibility line. The irreversibility temperature increases and the curvature of the irreversibility line changes the sign. It is accompanied by an enhancement of $j_{\mathrm{c}}$ and a decrease in the normalized relaxation rates [44]. Neutron irradiation experiments give more complicated results [45]. Diflerent shifts of the irreversibility 
line are observed, depending on the magnetic field orientation and values of neutron fluence. Neutron irradiation causes drastic reduction of the relaxation rates for temperatures up to $50 \mathrm{~K}$. At higher temperatures an increase is observed, in contrast to the behavior in the nonirradiated state [46].

The difference between effects of proton, heavy ion and neutron irradiation of HTSC can be presumably attributed to the different nature of the radiation induced defects. For protons, only small clusters of one or a few displacements are produced, therefore their nature is similar to the defects already existing in nonirradiated samples. They seem to be able to enhance $j_{\mathrm{c}}$ because of a larger number of defects, but not to slift the irreversibility line because of similar pinning potential in comparison with that existing originally. In the case of heavy ions and neutrons, large defects introduced by irradiation have pinning potentials different from existing initially. Therefore, they are able to enhance $j_{c}$ and shift the irreversibility line simultaneously. The effects of fast neutron irradiation on various types of ITSC materials are discussed in detail below.

\section{Influence of fast neutron irradiation on propertics of various types of HTSC}

The measurements discussed below concern the samples irradiated at room temperature. The values of fluence were estimated with an accuracy of $10 \%$ and they correspond to the neutron energies above $1 \mathrm{MeV}$. For a comparison with the experiments in which fluence is estimated for neutrons with energy $E>0.1 \mathrm{MeV}$ or $E>0.5 \mathrm{MeV}$ our value of fluence for $E>1 \mathrm{MeV}$ should be multiplied by 2.20 or 1.44 , respectively.

The majority of experiments, at least at early stage of investigations of IITSC, were done with sintered materials. Therefore, we start the discussion with ceramic samples.

\subsection{Ceramic samples}

In the case of ceramics we can distinguish two critical currents - the intragrain critical current which corresponds to the critical current of single crystals (bulk or thin film) and the intergrain critical current limited by the grains connections. It has to be bome in mind that an increase in the intragrain critical current (as deduced from magnetic measurements) after neutron irradiation is not accompanied by an increase in the intergrain current, determined from transport $j_{\mathrm{c}}$ measurements. This behavior is a consequence of an enhancement of flux pinning inside grains, associated in the same time with damages of intergrain junctions. Magnetic measurements of irradiated ceramics can tell us to what extent the intragrain $j_{\mathrm{c}}$ can be enhanced and what changes on $j_{\mathrm{c}}(T, B)$ can occur. They can also give us information on changes of $T_{\mathfrak{c}}$, of the normal state resistance as well as of the irreversibility line. Such studies enable one to establish the general features of the response of IITSC compounds to neutron irradiation. However, of course the anisotropy of the measured properties can not be inferred from these experiments.

Studies of neutron irradiation effects on ceramics have been carried out, by several groups of researchers, on different IITSC compounds, namely $\mathrm{LaSrCuO}$, 
e.g. Ref. [47], $\mathrm{YBaCuO}$, e.g. Ref. [35, 36, 48-51], $\mathrm{Bi}(\mathrm{Pb}) \mathrm{SrCaCuO}$, e.g. Ref. [52, 53] and also BaKBiO, e.g. Ref. [54]. In the present paper, on the base of the earlier works, the influence of fast neutron irradiation on the magnetic and transport properties of $\mathrm{YBa}_{2} \mathrm{Cu}_{3} \mathrm{O}_{7-x}$ and $\mathrm{Bi}_{0.7} \mathrm{~Pb}_{0.3} \mathrm{SrCaCu}_{1.8} \mathrm{O}_{x}$ is summarized. Also the comparison of irradiation eflects on the $60 \mathrm{~K}$ - and the $90 \mathrm{~K}$-phase of $\mathrm{YBaCuO}$ reported in Ref. [55], is discussed. In this case, because of the essential role of the oxygen in $\mathrm{YBaCuO}$ compounds, the comparative studies of radiation effects in both phases should be carried out.

The influence of irradiation on $T_{c}$ for the $90 \mathrm{~K}$-phase of $\mathrm{YBaCuO}$ and $110 \mathrm{~K}$-phase of $\mathrm{BiPbSrCaCuO}$ samples was studied in rather wide range of fluences, from $4.0 \times 10^{16}$ up to $3.3 \times 10^{18} \mathrm{n} / \mathrm{cm}^{2}(\mathrm{YBaCuO})$, and from $3.3 \times 10^{16} \mathrm{up}$ to $3.0 \times 10^{18} \mathrm{n} / \mathrm{cm}^{2}(\mathrm{BiPbSrCaCuO})$. Critical temperature drops more abruptly for $\mathrm{BiPbSrCaCuO}$ system (for a nonirradiated sample $T_{\mathrm{c}}=108 \pm 1 \mathrm{~K}$ ) than for $\mathrm{YBaCuO}\left(T_{\mathrm{c}}=92.3 \pm 0.5 \mathrm{~K}\right)$. The decrease in $T_{\mathrm{c}}$ is about $10.3 \mathrm{~K}$ per $10^{18} \mathrm{n} / \mathrm{cm}^{2}$ and about $2.4 \mathrm{~K}$ per $10^{18} \mathrm{n} / \mathrm{cm}^{2}$ for $\mathrm{Bi}$ - and Y-base materials, respectively. In the case of $60 \mathrm{~K}$-phase of $\mathrm{YBaCuO}$ the range of applied fuences was narrower $\left(5.0 \times 10^{16}\right.$ to $\left.1.5 \times 10^{17} \mathrm{n} / \mathrm{cm}^{2}\right)$ and it secms that $T_{\mathrm{c}}$ drops quicker than for the $90 \mathrm{~K}$-phase.

The lower critical field, determined from a linear part of $M(H)$ at $4.2 \mathrm{~K}$, decreases with fluence both for $\mathrm{YBaCuO}$ and $\mathrm{BiPbSrCaCuO}$ samples. In the first case, $H_{\mathrm{c} 1}$ equals $380 \pm 50$ Oe for a nonirradiated sample and drops down to $210 \pm 50$ Oe after irradiation with a fluence of $8.7 \times 10^{17} \mathrm{n} / \mathrm{cm}^{2}$. For $\mathrm{BiPbSrCaCuO}, H_{\mathrm{c} 1}$ equals $600 \pm 90$ Oe before irradiation and decreases to $300 \pm$ 50 Oe after irradiation with a fluence of $3.0 \times 10^{18} \mathrm{n} / \mathrm{cm}^{2}$. This behavior was confirmed later by magnetooptic measurements carried out on $\mathrm{Bi}_{2} \mathrm{Sr}_{2} \mathrm{CaCu}_{2} \mathrm{O}_{x}$ single crystals $[56,57] . H_{\mathrm{c} 1}$ is related to the penetration depth, $\lambda$, and to the correlation length, $\xi$, through the formula

$$
H_{\mathrm{c} 1}=\Phi_{0} \ln \kappa / 4 \pi \lambda,
$$

where $\kappa=\lambda / \xi$. Taking into account the clianges of $H_{\mathrm{c} 1}$ after neutron irradiation one could not exclude a priori the changes in the parameters $\lambda$ and $\xi$. Studies of irradiation influence on the penetration depth $\lambda$ are currently under way in our group. Ilowever, it is also possible that instead of $H_{\mathrm{c} 1}$ the surface barrier (Bean-Livingston barrier, see Ref. [58]) is affected by irradiation. This problem was discussed for example by Konczykowski [59].

The influence of irradiation on the intragrain critical current density could be easily inferred from $M(H)$ hysteresis loops. However, an accurate determination of the absolute values of $j_{\mathrm{c}}$ is difficult for ceramic samples, because the exact information on the sizes and shapes of grains (diameters of supercurrents loops) would be needed. The sizes of the grains were not controlled in our processing technology, so we could use only approximate mean diameters. In the case of ceramics, the data could be analyzed only in terms of the isotropic Bean model, which introduces additional uncertainties because of the anisotropic nature of these compounds. The most reasonable way of estimation of irradiation influence is to compare the widths of the hysteresis loops before and after irradiation. $\Delta M^{\mathrm{irr}} / \Delta M^{\text {nonirr }}$ are proportional to the corresponding fractional change of $j_{c}$, i.e. $j_{c}^{\text {irr }} / j_{c}^{\text {nonirr }}$. Comparison of 
the influence of irradiation on the $90 \mathrm{~K}$-phase of $\mathrm{YBaCuO}$ and on $\mathrm{BiPbSrCaCuO}$ ceramics at different temperatures is shown in Table.

TABLE

The influence of irradiation on $T_{c}$ and the intragrain critical currents measured at $4.2,42$ and $77 \mathrm{~K}$ in the magnetic field of $10 \mathrm{kOe}$ for polycrystalline $\mathrm{YBaCuO}$ and $\mathrm{BiPbSrCaCuO}$ systems.

\begin{tabular}{|c|c|c|c|c|c|}
\hline \multicolumn{6}{|c|}{$\mathrm{YBa}_{2} \mathrm{Cu}_{3} \mathrm{O}_{7-x}$} \\
\hline \multirow[t]{2}{*}{$T(\mathrm{~K})$} & \multicolumn{5}{|c|}{ fluence $\left(10^{18} \mathrm{n} / \mathrm{cm}^{2}\right)$} \\
\hline & 0.18 & 0.37 & 0.61 & 2.7 & 3.3 \\
\hline 4.2 & 1.8 & 1.8 & 2.0 & 1.6 & 1.7 \\
\hline$j_{\mathrm{c}}^{\text {irr }} / j_{\mathrm{c}}^{\text {nonirr }} 42$ & 7.0 & 6.4 & 11.7 & 5.1 & 5.7 \\
\hline 77 & 9.9 & 5.9 & 9.7 & 0.4 & 0.2 \\
\hline$\Delta T_{\mathrm{c}}(\mathrm{K})$ & -0.9 & -1.4 & -2.2 & -6.6 & -8.4 \\
\hline \multicolumn{6}{|c|}{$\mathrm{Bi}_{0.7} \mathrm{~Pb}_{0.3} \mathrm{SrCaCu}_{1.8} \mathrm{O}_{x}$} \\
\hline \multirow[t]{2}{*}{$T(\mathrm{~K})$} & \multicolumn{5}{|c|}{ fluence $\left(10^{18} \mathrm{n} / \mathrm{cm}^{2}\right)$} \\
\hline & 0.033 & 0.16 & 0.4 & 0.66 & 3.0 \\
\hline 4.2 & 1.2 & 1.8 & 2.0 & 1.9 & 0.9 \\
\hline$j_{\mathrm{c}}^{\text {irr }} / j_{\mathrm{c}}^{\text {nonirr }} 42$ & 2.0 & 7.6 & 20.5 & 13.0 & 0.5 \\
\hline$\Delta T_{\mathrm{c}}(\mathrm{K})$ & 0 & -3.0 & -4.1 & -6.5 & -31.0 \\
\hline
\end{tabular}

According to above discussion one can make only a rough comparison of $j_{\mathrm{c}}$ 's of these two compounds by assuming that the mean diameter of the supercurrent loops is the same in each case. For instance, if one assumes an average grain size of $10 \mu \mathrm{m}$ for the $90 \mathrm{~K}$-phase of $\mathrm{YBaCuO}$ samples irradiated with fluence of $6.1 \times 10^{17} \mathrm{n} / \mathrm{cm}^{2}, j_{\mathrm{c}}$ equals $4.2 \times 10^{6}$ and $8.4 \times 10^{4} \mathrm{~A} / \mathrm{cm}^{2}$ at 4.2 and $77 \mathrm{~K}$, respectively. It should be pointed out that in contrast to the $90 \mathrm{~K}$-phase of $\mathrm{YBaCuO}$, in the case of $\mathrm{BiPbSrCaCuO}$ samples, at $77 \mathrm{~K}$, even after irradiation a reversible behavior is observed in the hysteresis loops in higher fields. The similar situation is observed at $40 \mathrm{~K}$ for the $60 \mathrm{~K}$-phase of $\mathrm{YBaCuO}$. In addition, in the case of $60 \mathrm{~K}$-phase critical current density decreases more quickly witl temperature and field in comparison with the $90 \mathrm{~K}$-phase. This is in agreement with the fact that the irreversibility line in the $60 \mathrm{~K}$-phase is shifted to the region of lower fields and temperatures in comparison with the $90 \mathrm{~K}$-phase. This problem and the influence of irradiation on the position of irreversibility line are discussed below.

One could notice that the influence of irradiation is rather similar in the case of the $\mathrm{BiPbSrCaCuO}$ and $90 \mathrm{~K}$-phase of $\mathrm{YBaCuO}$. An increase in $j_{\mathrm{c}}$ is rather significant (for fluences up to $6.6 \times 10^{17} \mathrm{n} / \mathrm{cm}^{2}$ ), especially at higher temperatures and lower fields. In another experiment [55], the $60 \mathrm{~K}$ - and $90 \mathrm{~K}$-phase of $\mathrm{YBaCuO}$ ceramics have been subjected to fast neutron irradiation with the fluences of $0.35,0.76$ and $1.05 \times 10^{17} \mathrm{n} / \mathrm{cm}^{2}$. The effects of irradiation were found to be significantly different in the two phases. Whereas the enhancement of $j_{\mathrm{c}}$ was 
very pronounced in the $90 \mathrm{~K}$-phase (even up to the factors of 40 at $77 \mathrm{~K}$ and in $10 \mathrm{kOe}$ ), the increase in $j_{\mathrm{c}}$ was rather moderate in the $60 \mathrm{~K}$-phase (factors of 1.5 to 5) and only weakly temperature and field dependent. The influence of irradiation on the irreversibility line in the $60 \mathrm{~K}$ - and the $90 \mathrm{~K}$-phase of $\mathrm{YBaCuO}$ was reported in Ref. [55]. Irreversibility lines were studied by means of ac-susceptibility measurements $(1 \mathrm{Oe}, \nu=21 \mathrm{IIz}$ ). Although the irreversibility temperature in constant field is usually defined through the peak in the imaginary component of susceptibility $\chi^{\prime \prime}$, in the case of discussed measurements the tangents of real component $\chi^{\prime}$ were chosen due to the following reasons. In ceramics the $\chi^{\prime \prime}$ component usually shows two peaks which are related to the intra- and the intergrain currents, respectively. After irradiation, due to destruction of weak links between grains, the intragrain peak is masked by the continuously broadened intergrain peak. On the other hand, the $\chi^{\prime}$ signal remains reasonably sharp and could be used for determination of a position of the irreversibility line with a sufficient accuracy. The results both for the $60 \mathrm{~K}$ - and for the $90 \mathrm{~K}$-phase of $\mathrm{YBaCuO}$ are shown in Fig. 1. Following neutron irradiation a large shift of irreversibility line to higher fields and

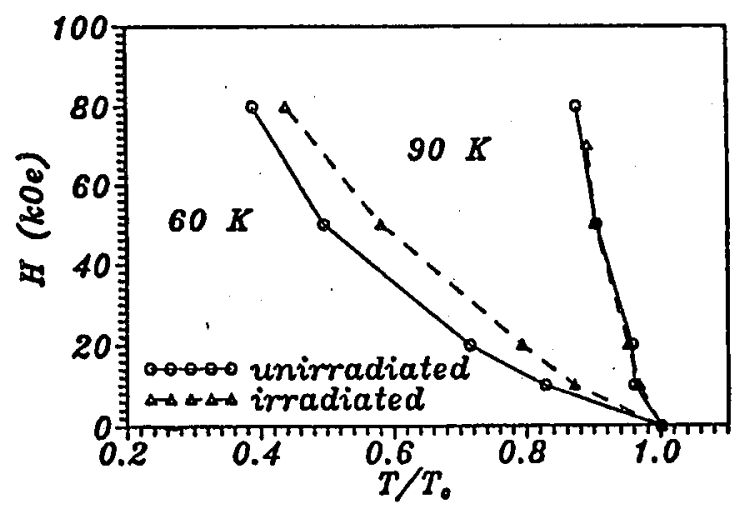

Fig. 1. Irreversibility lines for the $60 \mathrm{~K}$ - and the $90 \mathrm{~K}$-phase of polycrystalline $\mathrm{YBa}_{2} \mathrm{Cu}_{3} \mathrm{O}_{7-\delta}$ before and after fast nentron irradiation with the fluence of $0.76 \times 10^{17} \mathrm{n} / \mathrm{cm}^{2}$.

temperatures in the $60 \mathrm{~K}$-phase is observed, whereas this effect is very small in the $90 \mathrm{~K}$-phase. Comparison of the influence of irradiation on increase in $j_{\mathrm{c}}$ and position of the irreversibility line shows that the shift of the irreversibility line is not correlated with the enhancement of the critical current. Most likely it means that defects created by irradiation have the pinning potentials which are similar to the potentials of defects existing initially in the case of the $90 \mathrm{~K}$-phase, and are greater than potentials of initial defects in the case of the $60 \mathrm{~K}$-phase. The different response of both phases to the same fluences means that the same defects introduced into the $60 \mathrm{~K}$-phase are not eflicient pinning centers, whereas they are efficient in the case of the $90 \mathrm{~K}$-phase. This fact can indicate that the coherence length, $\xi$, in the $60 \mathrm{~K}$-phase is greater than in the $90 \mathrm{~K}$-phase.

The transport properties are strongly affected by irradiation. In this pa- 
per main conclusions will be discussed only, more detailed studies can be found in the previous papers $[36,60]$. For $\mathrm{YBaCuO}$ and $\mathrm{BiPbSrCaCuO}$ ceramics the following features are common. The normal state resistance, $R_{\mathrm{n}}$, systematically increases after irradiation. For example, in the case of $\mathrm{YBaCuO}$ samples room temperature resistivity increases from $2.1 \Omega \cdot \mathrm{cm}$ (the nonirradiated sample) up to $6.6 \Omega \cdot \mathrm{cm}$ for a sample irradiated with the fluence of $8.7 \times 10^{17} \mathrm{n} / \mathrm{cm}^{2}$. Simultaneously, temperature dependence of $R_{n}$ is more flat after irradiation. The ratio $R_{\mathrm{n}}(300 \mathrm{~K}) / R_{\mathrm{n}}(100 \mathrm{~K})$ is equal to 2.5 for nonirradiated samples and equal to 1.5 after irradiation with $8.7 \times 10^{17} \mathrm{n} / \mathrm{cm}^{2}$. Since the Josephson coupling energy, $E_{\mathrm{j}}$, is inversely proportional to the normal state resistivity, the increase in $R_{\mathrm{n}}$ is connected with the decrease in the crilical temperature as determined from the transport measurements. The zero resistance of a sample occurs when the Josephson coupling energy is comparable or greater than the thermal energy. Thus, for lower values of $E_{\mathrm{j}}$, the condition $E_{\mathrm{j}} \geq k_{\mathrm{B}} T$ is fulfilled at lower temperatures.

The most important result of the transport measurements is the direct evidence that the intergrain critical current decreases with increasing fluence as the consequence of a damage of the weak links by irradiation. The field dependence of $j_{\mathrm{c}}$ shows a remarkable decrease even in low magnetic fields. For example, in Fig. 2 for $\mathrm{BiPbSrCaCuO}$ samples a decrease in $j_{\mathrm{c}}$ of about one order of magnitude between zero field and $5 \mathrm{Oe}$ at $77 \mathrm{~K}$ is shown.

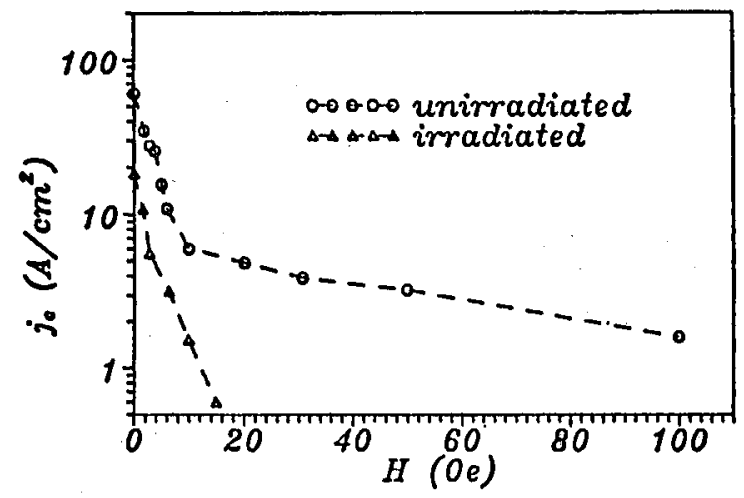

Fig. 2. The field dependence of the transport critical current of polycrystalline BiPb$\mathrm{SrCaCuO}$ at $77 \mathrm{~K}$ for a nonirradiated sample (circles) and for a sample irradiated with the neutron fluence of $4 \times 10^{17} \mathrm{n} / \mathrm{cm}^{2}$ (triangles).

An attempt was made to check the influence of fast neutrons on structural properties of $\mathrm{YBa}_{2} \mathrm{Cu}_{3} \mathrm{O}_{x}$ ceramics irradiated with the fluence between $4.0 \times 10^{16}$ and $8.7 \times 10^{17} \mathrm{n} / \mathrm{cm}^{2}$ [61]. Investigations of the crystal structure by X-ray and neutron powder diffraction show as an essential change a linear increase in the lattice constants $a, b$ and $c$ for small values of fluences and a decrease for higher fluences. A nearly isotropic variation of all three lattice parameters was observed for fluences below $1.2 \times 10^{17} \mathrm{n} / \mathrm{cm}^{2}$. Any other changes of the microscopic crystallographic parameters (occupancies and positional parameters) were noticed to be 
smaller than the statistical errors or the systematic differences between the X-ray and the neutron results.

As it was mentioned above, the itradiation causes opposite effects on the intra- and intergrain propertics of IITSC ceramics. It seems that one could find a range of neutron fluences where it is possible to enhance the intragrain critical currents up to about one order of magnitude or more (in fields of about $10 \mathrm{kOe}$ ) without significant drop of the critical temperature. On the other hand, radiation damages the weak links, what is evidenced by a reduction of the transport critical current and an increase in the normal state resistivity. Therefore from the point of view of applications the only perspective materials are those in which critical current is not limited by a field induced decoupling of the weak links.

It should to be mentioned that $\mathrm{Ba}_{1-x} \mathrm{~K}_{x} \mathrm{BiO}_{3}$ [54] compounds behave differently from other IITSC superconductors, since the intragrain critical currents are not affected by irradiation up to the fluence of $1.4 \times 10^{18} \mathrm{n} / \mathrm{cm}^{2}$.

\subsection{Mell-lextured malerials}

A few papers concerning the effect of fast neutron irradiation of melt-textured samples, have been published. Küpfer et al. [62] have investigated $\mathrm{YBaCuO}$ samples prepared by Salama et al. [25]. They found that the intragrain $j_{\mathrm{c}}$ increases after irradiation with the fluence of $10^{17} \mathrm{n} / \mathrm{cm}^{2}(E>1 \mathrm{MeV})$ by a factor of 4 reaching value of $2.7 \times 10^{4} \mathrm{~A} / \mathrm{cm}^{2}$ when the magnetic field was perpendicular to the $a-b$ plane and $5.3 \times 10^{3} \mathrm{~A} / \mathrm{cm}^{2}$ for the field parallel to this plane at $77 \mathrm{~K}$ and in $20 \mathrm{kOe}$. They have also studied magnetization relaxation and the position of the irreversibility line. The analysis of the relaxation data showed an increase in activation energy by a factor of about 1.5 at low temperatures and a decrease at high temperatures. Significant shifts of the reversibility lines for the magnetic field perpendicular and parallel to $a-b$ were noticed. Ifor et al. [63] investigated $\mathrm{YBaCuO}$ samples, obtained with some modification of the technique proposed by Salama et al. [25], irradiated up to the fluence of $6.0 \times 10^{17} \mathrm{n} / \mathrm{cm}^{2}$. They observed an increase in the magnetic intragrain $j_{\mathrm{c}}$ up to $2 \times 10^{5} \mathrm{~A} / \mathrm{cm}^{2}$ at $77 \mathrm{~K}$ and in $10 \mathrm{kOe}$. They have also found that thermal annealing up to $600^{\circ} \mathrm{C}$ recover the nonirradiated state.

We have investigated melt-textured samples prepared by powder melting process (PMP) $[27,64]$. In this approach instead of $\mathrm{YBa}_{2} \mathrm{Cu}_{3} \mathrm{O}_{7-x}$ powders of $\mathrm{Y}_{2} \mathrm{BaCuO}_{5}$ and $\mathrm{BaCuO}_{2}$ are used as precursors. After a careful mixing of these powders (homogeneous distribution of $\mathrm{Y}_{2} \mathrm{BaCuO}_{5}$ particles turns out to be very crucial for obtaining textured samples of high quality) they are heated to $1040^{\circ} \mathrm{C}$ and then cooled down. The best samples obtained with this method exhibit $j_{\mathrm{c}}$ of about $70000 \mathrm{~A} / \mathrm{cm}^{2}$ at $77 \mathrm{~K}$ and in $10 \mathrm{kOe}$ and $35000 \mathrm{~A} / \mathrm{cm}^{2}$ in $40 \mathrm{kOe}$ (pulse technique, pulse duration $5 \mathrm{~ms}$, voltage criterion $10 \mu \mathrm{V} / \mathrm{cm}$ ).

The observations of the microstructure of PMP samples were described by Zhou Lian and co-workers [61-66]. The morphology on the fresh fracture surface of the samples and microstructure were analyzed by SEM and TEM, respectively. According to SEM and TEM results PMP samples contain laycred grains of 123 phase - parallel to each other, and particles of non-superconducting 211 phase. 
No microcracks have been found in investigated samples. The thickness of grains is in the range of $0.1-3 \mu \mathrm{m}$. The layered grains have a perfect orientation and are not totally separated by the grain boundaries. The wide areas not containing any interfaces (a width of about $3 \mu \mathrm{m}$ ), where the twins pass through the boundaries, join the two 123 grains together and eliminate the grain boundaries. Remaining boundaries are very clean and low angle ones.

It is very important to know whether the weak links are really absent in the melt-textured samples and whether they are introduced during irradiation. The simplest way to check this is to find if the linear dependence between the width of the hysteresis loop (magnetization irreversibility) and the sample size (linear sizes of parallelepipeds in our case) is observed. This is a proof that the grains are not only connected better than in the case of regular ceramic but also that the grains cannot be disconnected by the magnetic field. In such samples one can believe that the weak links are absent in this sense that they do not limit $j_{\mathrm{c}}$ in the magnetic field. Thus, the critical current determined from the magnetization measurements corresponds to the current, which flows throughout the whole sample. IIowever, one cannot make a priori assumption that the lack of the weak links before irradiation assures their absence after irradiation. Following irradiation the regions on the borders of grains could be degraded. Additionally, after the increase of the intragrain current due to the created pinning centers inside the grains, regions on the border of grains may not carry highlier $j_{c}$. Therefore, it should be checked whether the weak links are absent in the irradiated samples.

Before irradiation the measurements of the magnetic critical current, $j_{\mathrm{cm}}$, and of the transport critical current, $j_{\mathrm{ct}}$, were carried out. There is a "technical" problem connected with the direct transport measurements of neutron irradiated samples. Because of high activation of silver during irradiation, silver contacts should be removed before irradiation. Putting new contacts, which should be hot pressed, leads to annealing of defects introduced during irradiation. Therefore, only the magnetic measurements of irradiated samples were possible at the moment. However, according to the arguments discussed below, we believe that it is possible to enhance the critical current density for the current flowing throughout the whole sample. We believe that it is possible to obtain values of the order of $10^{5} \mathrm{~A} / \mathrm{cm}^{2}$ at $77 \mathrm{~K}$ and in $10 \mathrm{kOe}$.

The values of $j_{\mathrm{ct}}$ determined by the pulse current method (pulse duration $5 \mathrm{~ms}$, voltage criterion $10 \mu \mathrm{V} / \mathrm{cm}$ ) were ranged for various samples between $3 \times 10^{3}$ and $7 \times 10^{4} \mathrm{~A} / \mathrm{cm}^{2}$ at $77 \mathrm{~K}$ and in $10 \mathrm{kOe}$. For the best sample, $j_{\mathrm{ct}}$ at $77 \mathrm{~K}$ and in $40 \mathrm{kOe}$ was $3.5 \times 10^{4} \mathrm{~A} / \mathrm{cm}^{2}$. The magnetic measurements were performed using the vibrating sample magnetometer in the magnetic field up to $16 \mathrm{kOe}$. Samples of two lengths were investigated: longer - of about $10 \mathrm{~mm}$ (which were measured by the transport technique also), and shorter - of about $6 \mathrm{~mm}$. Magnetic measurements of longer samples could be done only with magnetic field perpendicular to the longest sample size. In these measurements only the average critical current in two planes containing the transport current direction, $j_{\mathrm{cm} 1}$ and $j_{\mathrm{cm} 2}$, could be estimated. The comparison with $j_{\mathrm{ct}}$ gave reasonable agreement. For example, at $77 \mathrm{~K}$ and in $10 \mathrm{kOe}$, for the sample with $j_{\mathrm{ct}}=2 \times 10^{4} \mathrm{~A} / \mathrm{cm}^{2}$ the values of $j_{\mathrm{cm} 1}=1.2 \times 10^{4} \mathrm{~A} / \mathrm{cm}^{2}$ and $j_{\mathrm{cm} 2}=1.1 \times 10^{4} \mathrm{~A} / \mathrm{cm}^{2}$ were obtained. 
Two shorter samples were used to perform the measurements in three different orientations of the sample with respect to the magnetic field. At the beginning, the decrease in the magnetization irreversibility proportional to the reduction of the sample sizes was found for bulk PMP nonirradiated samples [67]. Next, we checked whether the weak links were not introduced during irradiation, as is presented below. The parallelepiped sample with initial dimensions $5.3 \times 1.6 \times 0.9 \mathrm{~mm}^{3}$ was irradiated by fast neutrons with the fluence of $1.32 \times 10^{17} \mathrm{n} / \mathrm{cm}^{2}(E>1 \mathrm{MeV})$. The measurements of hysteresis loop width were done on the sample with initial dimensions and then after reduction to $4.0 \times 1.2 \times 0.6 \mathrm{~mm}^{3}$ and $1.6 \times 1.0 \times 0.3 \mathrm{~mm}^{3}$, respectively. For each size measurements were performed with the magnetic field perpendicular to each sample plane at 60,77 and $85 \mathrm{~K}$. For all three orientations of the magnetic field roughly linear dependence of the hysteresis loop width on the sample size was found. The results of the experiment indicate that the weak links are not introduced during irradiation, and/or that the links existing between grains are not damaged due to irradiation.

These results were confirmed by microwa ve absorption in low magnetic fields. Although the mechanism of low-ficld microwave absorption (LFMA) is not clarified yet, it is generally accepted that important contribution to LFMA arises from the Josephson links with finite sizes, which are decoupled by magnetic fields (the swept external magnetic field as well as the modulation field), microwave current and thermal fluctuations $[68,69]$. LFMA signal was measured with a standard Xband electron spin resonance spectrometer. The observed LFMA signal was not resembling a typical one for polycrystalline superconductors. For ceramic samples one should observe very large LFMA, observable down to the lowest liquid helium temperatures. It means that the weak links remain really weak over whole temperature range where the superconductivity exists. In contrary, in melt-textured superconductors LFMA was observed only close to $T_{\mathrm{c}}$. LFMA signals in both samples, irradiated and nonirradiated, are similar one to another supporting our statement concerning insignificant number of the weak links in melt-textured superconductors.

The critical current density $j_{\mathrm{c}}$ was determined from the magnetization measurements, basing on the modified anisotropic Bean model $[6,33]$. Magnetic measurements of a cylindrical sample with the length $l$ about one order of magnitude higher than the diameter $d$ and with the $c$-axis perpendicular to the length of the sample were performed. The applied magnetic field was perpendicular to the length of the cylinder. For the magnetic field $I I$ perpendicular to the $a-b$ plane the critical current density in the $a-b$ plane $j_{c}^{a b, c}$ was calculated using the following formula:

$$
j_{\mathrm{c}}^{a b, c}=\frac{20 \Delta M_{a b}}{d(1-d / 3 l)}
$$

where $\Delta M_{a b}$ is the width of the hysteresis loop for $H$ perpendicular to the $a-b$ plane. The critical current density along $c$-axis was calculated using $j_{c}^{a b, c}$ and the width of the hysteresis loop for the magnetic field parallel to the $a-b$ plane. We 
have assumed $j_{\mathrm{c}}^{a b, a b}=j_{\mathrm{c}}^{a b, c}$ and we have applied the formulae [6]

$$
\Delta M_{\mathrm{c}}=\frac{j_{\mathrm{c}}^{\mathrm{c}, a b} l}{20}\left(1-\frac{l}{3 d} \frac{j_{\mathrm{c}}^{\mathrm{c}, a b}}{j_{\mathrm{c}}^{a b, a b}}\right)
$$

valid for $j_{\mathrm{c}}^{a b, a b} / j_{\mathrm{c}}^{\mathrm{c}, a b}>l / d$ and

$$
\Delta M_{\mathrm{c}}=\frac{j_{\mathrm{c}}^{\mathrm{ab}, a b} d}{20}\left(1-\frac{d}{3 l} \frac{j_{\mathrm{c}}^{a b, a b}}{j_{\mathrm{c}}^{c, a b}}\right)
$$

valid for $j_{\mathrm{c}}^{a b, a b} / j_{\mathfrak{c}}^{c, a b}<l / d$. The width of the hysteresis loop $\Delta M_{\mathrm{c}}$ for a field parallel to the $a-b$ plane is a function of both $j_{c}^{a b, a b}$ and $j_{c}^{c, a b}$ (principal axis components of critical current density). So, if $j_{\mathrm{c}}^{a b, a b}$ is known, the critical current density $j_{\mathrm{c}}^{c, a b}$ along $c$-axis is a solution of the quadratic equation (7) or the linear equation (8). Equations (7) and (8) are not generally applicable because $j_{c}^{a b, c} \leq j_{c}^{a b, a b}$. However, the maximal error seems to be less than $10 \%$ in our case, for estimation done with the assumption of the infinite $j_{\mathrm{c}}^{a b, a b}$ value.

The enhancement of the critical current density as a result of fast neutron irradiation was observed for all investigated fluences. The highest value of the critical current density, $j_{c}^{a b, c}=9.4 \times 10^{4} \mathrm{~A} / \mathrm{cm}^{2}$ at $77 \mathrm{~K}$ in $10 \mathrm{kOe}$, was obtained for the sample irradiated with the flucnce of $4.2 \times 10^{17} \mathrm{n} / \mathrm{cm}^{2}$ (before irradiation $j_{\mathrm{c}}^{a b, c}=2.8 \times 10^{4} \mathrm{~A} / \mathrm{cm}^{2}$ ) and $j_{\mathrm{c}}^{c, a b}=5.0 \times 10^{3} \mathrm{~A} / \mathrm{cm}^{2}$ (before irradiation $j_{\mathrm{c}}^{c, a b}=2.2 \times 10^{3} \mathrm{~A} / \mathrm{cm}^{2}$ ). The maximum enhancement of $j_{\mathrm{c}}$ in the full range of applied fluences was equal to about 4 . The enhancement is significant, taking into account a rather high value of critical current before irradiation.

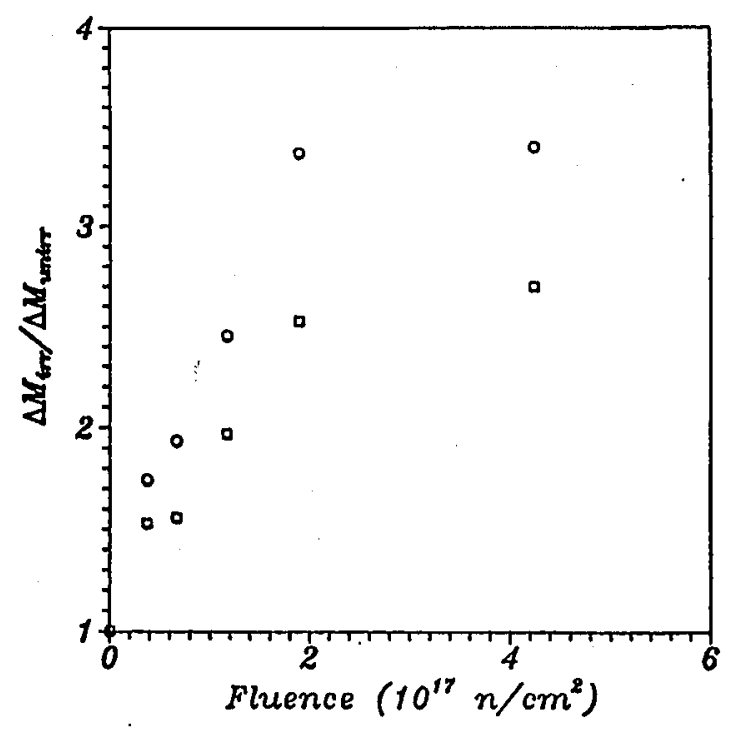

Fig. 3. Increase in the width of the liysteresis loop of the melt-textured $\mathrm{YBaCuO}$ as a result of fast neutron irradiation, measured at $77 \mathrm{~K}$ in $10 \mathrm{kOe}$ for the magnetic field perpendicular (circles) and parallel (squares) to the $a-b$ plane. 
In the investigated range of temperatures, the enhancement of the critical current was more pronounced for $H$ perpendicular to the $a-b$ plane in the whole range of applied fluences. The increase in the width of the hysteresis loop measured at $77 \mathrm{~K}$ in $10 \mathrm{kOe}$ is shown in Fig. 3 for both orientations of the field and for all applied fluences. We found that in the investigated range of temperatures $(T>60 \mathrm{~K})$ in the magnetic field of $10 \mathrm{kOe}$ anisotropy of critical current density increased after irradiation. This effect is in agreement with the results of Küpfer et al. [62]. However, it should be emphasized that at low temperatures, the opposite tendency was found in neutron irradiated YBCO single crystals. Sauerzopf et al. [70] found a pronounced decrease in anisotropy of $j_{c}$ at $5 \mathrm{~K}$ after irradiation. Apparently, these differences can be connected with the size of defects, which are effective pinning centers in low temperatures for two principal axis components of the critical current. At higher temperatures these defects can be still effective in the $a-b$ plane but not for the current flowing along $c$-axis. The anisotropy of critical currents is influenced by an interaction of the material properties anisotropy and the different pinning structures. Regarding this, a comparison between single crystals and melt-textured materials may lead to erroneous interpretations. The investigations of the infuence of irradiation on activation energy are necessary for a full understanding of the temperature dependence of the anisotropy of critical current. Such studies are currently being done.

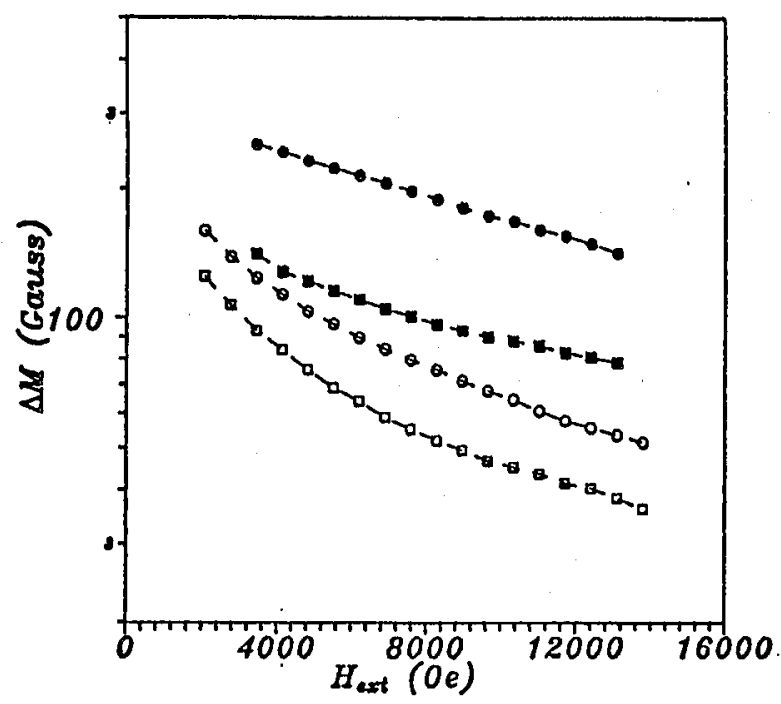

Fig. 4. Field dependence of the width of the hysteresis loop at $77 \mathrm{~K}$ for the magnetic field perpendicular and parallel to the $a-b$ plane for the melt-textured $\mathrm{YBaCuO}$ nonirradiated and irradiated with the fluence of $1.18 \times 10^{17} \mathrm{n} / \mathrm{cm}^{2}$ (open circles - a nonirradiated sample, $H$ perpendicular to the $a-b$ plane; open squares - a nonirradiated sample, $H$ parallel to the $a-b$ plane; full circles - an irradiated sample, $H$ perpendicular to the $a-b$ plane; full squares - an irradiated sample, II parallel to the $a-b$ plane). 


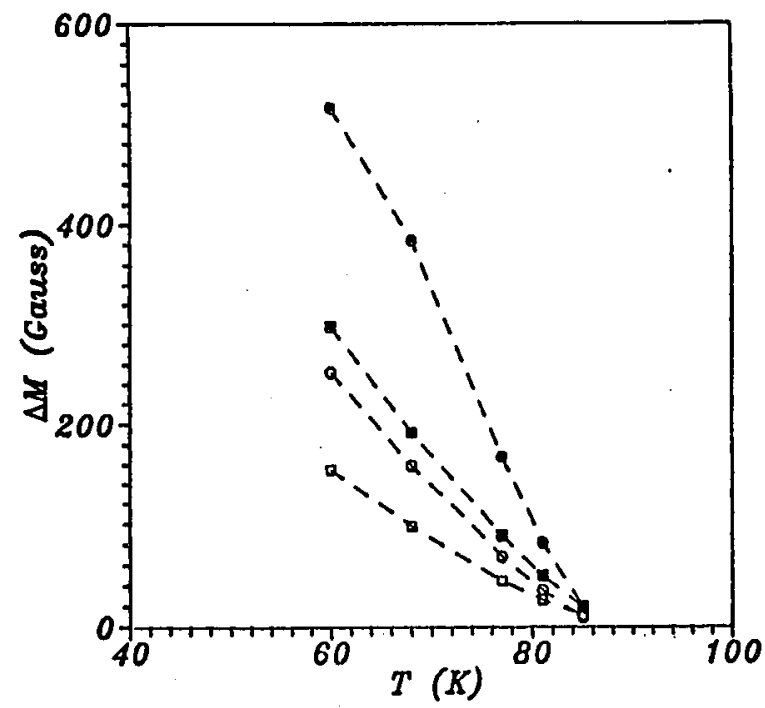

Fig. 5. Temperature dependence of the width of the hysteresis loop in $10 \mathrm{kOe}$ for the melt-textured $\mathrm{YBaCuO}$ nonirradiated and irradiated with the fluence of $1.18 \times 10^{17} \mathrm{n} / \mathrm{cm}^{2}$ (open circles - a nonirradialed sample, $I I$ perpendicular to the $a-b$ plane; open squares - a nonirradiated sample, $I$ parallel to the $a-b$ plane; full circles - an irradiated sample, $I I$ perpendicular to the $a-b$ plane; full squares - an irradiated sample, $H$ parallel to the $a-b$ plane).

The field dependence of critical current density after irradiation was improved only insignificantly or not improved at all, especially in higher fields (Fig. 4). It is in agreement with the results of Küpfer et al. [62]. The temperature dependence of $j_{\mathrm{c}}$ is not significantly affected by irradiation (Fig. 5). In single crystals, the increase in critical current was much more pronounced at higher temperatures [70]. Our experimental results can be explained by the existence of effective pinning centers in nonirradiated material, with as deep potentials as those of the radiation-induced defects. Such a conclusion could be confirmed by the study of the irreversibility line in nonirradiated and fast neutron irradiated samples.

More details concerning experiments discussed above are presented in the papers of Puźniak et al. [71, 72].

\section{Conclusions}

A deep knowledge of the role of defects in different types and forms of HTSC materials is one of the main targets to be pursued in IITSC research. Basic understanding of the physics concerning the competition between order and disorder in these materials as well as the devclopment of IITSC applications depend on our understanding the processes, which limit critical currents. Therefore, the introduction of defects into IITSC materials, by a controlled manner of irradiation, 
is an important tool to study and to develop possibilities of obtaining critical currents densities of the order of $10^{5} \mathrm{~A} / \mathrm{cm}^{2}$ at $77 \mathrm{~K}$. The study of the influence of neutron, proton, and heavy ion irradiation on magnetic, transport and structural properties of IITSC compounds should give an answer to the question to what extent the critical current density can be enhanced in IITSC. On the other hand, an attempt should be made to develop a consistent picture of the mechanism responsible for the observed phenomena. From the point of view of applications, the most promising are those materials, where $j_{c}$ is not limited by the weak links, i.e. high quality epitaxial thin films and melt-textured materials. As pointed out above, protons, neutrons and heavy ions produce defects of different kinds, which should have a different influence on the properties of the investigated compounds. The effect of irradiation is strongly dependent on the applied dose, on account of the concentration of the introduced defects. One can assume that for a particular type of material a kind of optimal radiation and an optimal range of doses could be found. It should be emphasized that irradiation with high doses leads to a significant drop in $T_{\mathrm{c}}$. For this reason, the applied doses should enable one to make a significant increase in $j_{\mathrm{c}}$ with only a moderate decrease in the critical temperature.

\section{Acknowledgments}

The authors would like to express their acknowledgments to Dr. K. Pytel and Dr. B. Pytel, from the Institute of Atomic Energy, Świerk, Poland, responsible for irradiation of the investigated samples. The author's would like to thank Prof. L. Dobrzyniski for critical reading the manuscript.

This work has been supported by the Committee for Scientific Research (KBN) under the Contract No. 204849101.

\section{References}

[1] A.M. Campbell, J.M. Evelts, Adv. Phys. 21, 199 (1972).

[2] M. Tachiki, S. Takahashi, Solid State Commun. 70, 291 (1989).

[3] J. Clem, Bull. Am. Phys. Soc. 35, 260 (1990).

[1] K. Watanabe, H. Yamane, II. Kurosawa, T. IIirai, N. Kobayashi, H. Iwasaki, K. Noto, Y. Muto, Appl. Pliys. Lett. 54, 575 (1989).

[5] A.P. Malozemoff, in: IIigh Temperature Superconducting Compounds II, Eds. S.H. Whang, A. Das Gupta, R.B. Laibowitz, TMS Publications, Warrendale (PA) 1990.

[6] E.M. Gyorgy, R.B. van Dover, K.A. Jackson, L.F. Schneemeyer, J.V. Waszczak, Appl. Phys. Lett. 55, 283 (1989).

[7] M. Tinkham, IEEE Trans. Mag. 27, 828×(1991).

[8] E.M. Brandt, Physica C 105, 1 (1992).

[9] P.W. Anderson, Phys. Rev. Lett. 9, 309 (1962).

[10] P.H. Kes, J. Aarts, J. van den Berg, C.J. van der Beek, J.M. Mydosh, Supercond. Sci. Technol. 1, 242 (1989).

[11] Y. Iye, T. Tamegai, II. Takeya, II. Takei, Jpn. J. Appl. Phys. 2, Lett. 26, L1057 (1987). 
[12] Y. Yeshurun, A.P. Malozemoff, Phys. Rev. Lett. 60, 2202 (1988).

[13] Y. Yeshurun, A.P. Malozemoff, F. Iloltzberg, T.R. Dinger, Phys. Rev. B 38, 11828 (1988).

[14] G. Blatter, V. Geslıkenbein, V. Vinokur, Phys. Rev. Lett. 66, 3297 (1991).

[15] A.C. Mota, G. Juri, P. Visani, A. Pollini, T. Teruzzi, K. Aupke, B. Hilti, Physica $C$ 185-189, 343 (1991).

[16] M. Suenaga, A.K. Gosh, Youwen Xu, D.O. Welch, Phys. Rev. Lett. 61, 1777 (1991).

[17] R.B. van Dover, E.M. Gyorgy, L.F. Schneemeyer, J.W. Mitchell, K.V. Rao, R. Puźniak, J.V. Waszczak, Nature 342, 55 (1989).

[18] M. Daeumling, J.M. Seuntjens, D.C. Larbalcstier, Nalure 346, 332 (1990).

[19] H. Küpfer, I. Apfelstedt, R. Flükiger, C. Keller, R. Meier-Hirmer, B. Runtsch, A. Turowski, U. Wiech, T. Wolf, Cryogenics 29, 268 (1989).

[20] Ch. Gerber, D. Anselmelli, J.G. Bednorz, J. Mannhliart, D.G. Schlom, Nature 350, 279 (1991).

[21] F. Chen, B. Zhang, R.S. Markiewicz, B.C. Giessen, Appl. Phys. Lett. 58, 531 (1990).

[22] S.L. Shinde, J. Morrill, D. Goland, D.A. Chance, T. McGuire, Phys. Rev. B 41, 8838 (1990).

[23] O.F. Schilling, Y. Yang, C.R.M. Grovenor, C. Beduuaz, Physica C 170, 123 (1990).

[24] S. Jin, J.H. Tiefel, R.C. Sherwood, R.B. van Dover, M.E. Davies, G.W. Kammlott, R.A. Fastnacht, Phys. Rev. B 37, 7850 (1988); S. Jin, T.II. Tiefel, R.C. Sherwood, M.E. Davies, R.B. van Dover, G.W. Kammlott, R.A. Fastnacht, H.D. Keith, Appl. Phys. Lelt. 52, 2074 (1988).

[25] K. Salama, V. Selvamanickam, L. Gao, K. Sun, Appl. Phys. Lett. 54, 2352 (1989).

[26] M. Murakami, M. Morita, K. Doi, K. Miyamoto, Jpn. J. Appl. Phys. 28, 1189 (1989).

[27] Zhou Lian, Zhang Pingxiang, Ji Ping, Wang Kequang, Wang Jingrong, Wu Xiozu, Supercond. Sci. Technol. 3, 490 (1990).

[28] T. Aselage, K. Keefer, J. Maler. Res. 3, 1279 (1989).

[29] M. Murakami, S. Gotoh, H. Fujimoto, K. Yamagucli, N. Koshizuka, S. Tanaka, Supercond. Sci. Teclinol. 4, S43 (1991).

[30] K. Sato, T. Hitaka, II. Mukai, M. Ueyama, N. Shibuta, T. Káato, T. Masuda, M. Nagata, K. Iwata, T. Mitsui, IEEE Trans. Mag. 27, 1231 (1991).

[31] L.D. Woolf, W.A. Raggio, F.E. Elsner, M.V. Fisher, R.B. Stephens, T.L. Figueroa, C.H. Shearer, J.D. Rose, K.M. Schaubel, R.A. Olstad, T. Ohkawa, D.M. Duggan, M. DiMartino, R.L. Fagaly, Appl. Phys. Lett. 58, 534 (1991).

[32] C.P. Bean, Rev. Mod. Phys. 36, 31 (1961).

[33] W.A. Fietz, W. Webb, Phys. Rev. 178, 657 (1969).

[34] H.W. Weber, G.W. Crabtree, in: Sludies of Iligh Temperalure Superconductors, Ed. A.V. Narlikar, Vol. 9, Nova Science Publishers, New York 1991, p. 37.

[35] H. Küpfer, I. Apfelstedt, W. Schauer, R. Flükiger, R. Meier-Hirmer, H. Wühl, H. Scheurer, Z. Phys. B 60, 167 (1987).

[36] P. Przyslupski, A. Wiśnicwski, S. Koleśnik, W. Dobrowolski, A. Pajączkowska, K. Pytel, B. Pytel, Physica C 153-155, 345 (1988). 
[37] K.V. Rao, R. Puźniak, D.-X. Chen, N. Karpe, M. Baran, A. Wiśniewski, K. Pytel, H. Szymczak, K. Dyrbye, J. Bottinger, Physica C 153-155, 347 (1988).

[38] R.L. Fleischer, II.R. Hart jr., K.W. Lay, F.E. Luborsky, Phys. Rev. B 40, 2163 (1989).

[39] Y.J. Zhao, J.R. Liu, R.L. Meng, W.K. Chu, Physica C 198, 256 (1992).

[40] M. Brezgunov, S. Belogurov, A. Petrov, A. Karpov, V. Kovalov, I. Kudrenitskis, Mater. Sci. For. 62-64, 169 (1990).

[41] V. Hardy, D. Groult, J. Provost, B. Raveau, Physica C 100, 289 (1992).

[42] L. Civale, A.D. Marwick, M.W. McElfresli, T.K. Worthington, A.P. Malozemoff, F.H. Holtzberg, J.R. Thompson, M.A. Irirk, Phys. Rev. Lelt. 65, 1164 (1990).

[43] M. Konczykowski, F. Rullier-Albenque, E.R. Yacoby, A. Shaulov, Y. Yeshurun, Phys. Rev. B 44, 7167 (1991).

[44] M. Konczykowski, F. Rullier-Albenque, Y. Yeshurun, E.R. Yacoby, A. Shaulov, P. Lejay, Plıysica C 185-189, 2317 (1991).

[15] H.W. Weber, Physica C 185-189, 309 (1991).

[46] J.G. Lensink, R. Griessen, II.P. Wiesinger, F.M. Sauerzopl, II.W. Weber, G.W. Crabtree, Physica C 185-189, 2287 (1991).

[47] S.T. Sekula, D.K. Christen, II.R. Kcrchner, J.R. Thompson, L.A. Boatner, B.C. Sales, Jpn. J. Appl. Phys. Suppl. 2C-3, 1185 (1987).

[48] K. Atobe, H. Yoshida, Pliys. Rcv. B 36, 7194 (1987).

[49] P. Müller, H. Gerstenberg, M. Fischer, W. Schindler, J. Ströbel, G. Saemann-Ischenko, H. Kammermeier, Solid State Comm. 65, 223 (1988).

[50] A. Wiśniewski, M. Baran, P. Przyslupski, II. Szymczak, A. Pajączkowska, B. Pytel, K. Pytel, Solid State Comm. 65, 577 (1988).

[51] B.A. Aleksashin, I.F. Berger, S.V. Verkhovskii, V.I. Voronin, B.N. Goshclitskii, S.A. Davydov, A.E. Karkin, V.L. Kozhevnikov, A.V. Mirmelshtein, K.N. Mikhaylov, V.D. Parhomenko, S.M. Cheshniskii, Plıysica C 153-155, 339 (1988).

[52] A. Wiśniewski, M. Baran, Z. Kozioł, P. Przysłupski, J. Picchota, R. Puźniak, A. Pajączkowska, M. Pękala, B. Pytcl, K. Pytel, Physica C 170, 333 (1990).

[53] Y.H. Herr, K.H. Lee, C.J. Kim, II.G. Lee, C.T. Kim, G.W. Hong, D.Y. Won, Jpn. J. Appl. Phys. 28, L1561 (1989).

[54] J.D. Thompson, J.R. Cost, G.II. Kwei, K.C. Ott, Physica C 169, 265 (1988).

[55] A. Wiśniewski, R.M. Sclıalk, II.W. Weber, M. Reissner, W. Steiner, J. Górecka, Physica C 185-189, 2211 (1991).

[56] R. Szymczak, M. Baran, K. Piotrowski, II. Szymczak, in: Proc. Internat. Workshop on Critical Current Limitations in IIigh Temperalure Superconductors, Eds. M. Baran, W. Gorzkowski, II. Szymczak, World Scientific, Singapore 1992, p. 246.

[57] K. Piotrowski, R. Szymczak, M. Baran, II. Szymczak, J. Magn. Magn. Mater. 104107, 483 (1992).

[58] M.W. McElfresh, Y. Yeshurun, A.P. Malozcmoff, F. Holtzberg, Physica A 168, 308 (1990).

[59] M. Konczykowski, in: Proc. Inlernat. Workshop on Critical Current Limitations in High Temperature Superconductors, Eds. M. Baran, W. Gorzkowski, H. Szymczak, World Scientific, Singapore 1992, p. 152. 
[60] P. Przyshupski, A. Wiśniewski, W. Dobrowolski, A. Pajączkowska, K. Pytel, B. Pytel, in: Physics and Materials Science of HTSC, Eds. R. Kossowsky, S. Methfessel, D. Wohlleben, Kluwer Academic Press, Dordreclit 1990, p. 641.

[61] J. Waliszewski, N.II. Anderson, L. Dobrzyniski, J. Ihringer, B. Lebech, W. Prandl, A. Wiśniewski, Physica C 160, 189 (1989).

[62] H. Küpfer, C. Keller, R. Meier-Hirmer, K. Salama, V. Selvamanickam, G.P. Tartaglia, IEEE Trans. Magn. MAG-27, 1369 (1991).

[63] P.H. Hor, Z.J. Huang, L. Gao, R.L. Meng, Y.Y. Xue, C.W. Chu, Y.C. Jean, J. Farmer, Mod. Phys. Lelt. B 4, 703 (1990).

[64] Zhou Lian, in: Lectures on IIigh Temperalure Superconductivity, Eds. Z.Z. Gan, R.S. Han, S.S. Xie, G.Z. Yang, Z.X. Zhao, World Scientific, Singapore 1991, p. 51.

[65] Wang Keguang, Zhou Lian, Zhang Pingxiang, Wang Shuqian, Wang Jingrong, Ji Ping, presented at ICMC-1991, Inntsville, Alabama, U.S.A., June 10-14, 1991, to be published in Advances in Cryogenic Engineering Materials.

[66] Zhou Lian, Zhang Pingxiang, Wang Ḱcguang, Wang Jingrong, Du Zehua, Ji Ping, presented at ICMC-1991, IIuntsville, Alabama, U.S.A., June 10-14, 1991, to be published in Advances in Cryogenic Engineering Materials.

[67] A. Wiśniewski, R. Puźniak, M. Gutowski, M. Baran, H. Szymczak, Wang Jingrong, Zhang Pingxiang, Ji Ping, Wang Keguang, Zhou Lian, Physica $C$ 185-189, 2503 (1991).

[68] R. Durny, J. Hantala, S. Ducharme, B. Lce, D.G. Symko, P.C. Taylor, D.C. Zheng, J.A. Xu, Phys. Rev. B 36, 2361 (1987).

[69] J. Stankowski, P.K. Kahol, N.S. Dalat, J.S. Moodera, Phys. Rev. B 36, 7526 (1987).

[70] F.M. Sauerzopf, H.P. Wiesinger, W. Kritscha, II.W. Weber, G.W. Crabtree, J.Z. Liu, Phys. Rev. B 43, 3091 (1991).

[71] R. Puźniak, A. Wiśniewski, II. Szymczak, Jingrong Wang, Pingxiang Zhang, Lian Zhou, Appl. Phys. Lelt. 61, 2102 (1992).

[72] R. Puźniak, A. Wiśniewski, M. Baran, II. Szymczak, Zlang Pingxiang, Wang Jingrong, Zhou Lian, K. Pytel, B. Pytel, Cryogenics 33, 261 (1993). 